\title{
Perspectivism and the epistemology of experimentation
}

\section{From the evaluation to the production of reliable experiments}

\section{Jan Potters}

Received: date / Accepted: date

\begin{abstract}
My aim in this paper is to propose a way to study the role of perspectives in both the production and justification of experimental knowledge claims. My starting point for this will be Anjan Chakravartty's claim that Ronald Giere's perspectival account of the role of instruments in the production of such claims entails relativism in the form of irreducibly incompatible truths. This led Michela Massimi to argue that perspectivism, insofar as it wants to form a realist position, is only concerned with the justification of such claims: whether they are produced reliably is, on her view, a perspective-independent fact of the matter. Following a suggestion by Giere on how scientists handle incompatible experimental results, I will then argue that Massimi's perspectivism can be extended to also cover the production of such claims, without falling into relativism. I will elaborate this suggestion by means of Uljana Feest's work on how scientists handle incompatible experimental results. I will argue that, if we reconceptualize perspectives
\end{abstract}

The author would like to acknowledge the Research Foundation - Flanders (FWO) as funding institution. Part of this paper was written during a stay as a visiting researcher at the University of Edinburgh with Michela Massimi's ERC-project Perspectival Realism: Science, Knowledge and Truth from a Human Vantage Point. Jan Potters

Center for Philosophical Psychology, Department of Philosophy, Rodestraat 14, 2000 Antwerpen, Belgium

E-mail: Jan.Potters@UAntwerpen.be 
as embodied and situated ways of going about in experimentation that can be made explicit through interpretation, we can obtain a fruitful understanding of the role of perspectives in both the production and justification of experimental knowledge. While this role is primarily exploratory, it can still allow for a substantial form of realism.

Keywords Perspectivism · Realism · Experimentation · Experimenters' Regress · Scientific Practice

\section{Introduction}

In his (2006) book, Ronald Giere argued that the way in which scientists produce scientific knowledge claims about particular target systems should urge us to become perspectivists with respect to these claims. By this, he meant that we should take these claims as telling us not what the target systems are like in themselves, but rather what they are like from the perspective that scientists used to formulate the claim. Giere argued that this could be seen, for example, in cases of experimental detections, i.e. the production of representations of particular characteristics of a target system by means of instruments. The reason for this is the role inherently played by these instruments: they mediate the information that we can obtain from the target system. In his (2010) article, Anjan Chakravartty argued, however, that insofar as such a perspectivism with respect to experimental detections is taken as a philosophically substantial position, it collapses into relativism, since it entails that we can only produce perspective-dependent irreducibly incompatible truths, not discover perspective-independent ones. This has led Michela Massimi (2012) to develop a version of perspectivism according to which only the claim's justification, i.e. our belief in the reliability of the instrument that produced the representation, is perspectivedependent. Whether or not the claim is true, i.e. whether it has been produced by a reliable 
experiment, is, on the other hand, something that is determined by a perspective-independent fact of the matter.

This move by Massimi entails that perspectives only play a role in how experimental knowledge claims get justified. They play no role of significance in, and hence perspectivism cannot tell us anything about, how these knowledge claims get produced. The reason for this is that both Chakravartty and Massimi assume that if perspectives also play a role in the production of experimental knowledge claims, there is no way to establish, in a perspective-independent way, that an experiment has operated reliable, which would entail a perspective-dependent, and hence relativistic, account of truth. It is my aim to argue here that we can in fact allow for perspectives playing a role in the production of experimental knowledge claims without ending up with relativism. I will do this by elaborating further a suggestion by Giere about how scientists address and overcome situations in which they are confronted with instruments that produce incompatible experimental results, which are the kinds of situations that perspectivism, according to Chakravartty, cannot handle without falling into relativism.

My starting point for this elaboration will be Uljana Feest's (2016) work on experimenters' regress situations, which are situations in which scientists are confronted with such incompatibilities, in the form of disagreements about the reliability of particular experiments. Feest's analysis of how scientists handle such situations, I will then argue, offers us a way to understand how incompatibilities between perspectives can be addressed, if we reconceptualize perspectives as embodied and situated ways of going about in experimental practice that scientists try to explicate through a process of interpretation. In this way, I will then argue, we can obtain a perspectivism that is broader in scope than Massimi's, since it covers not only justification and the evaluation of an experiment's reliability, but also its production. While this will require us to reconceptualize Massimi's epistemology of experimentation, focusing more on its exploratory 
aspects, the perspectivism presented here can still be taken to allow for realism with respect to experimental knowledge claims.

My concern in this paper will be with how we are to understand the role played by perspectives in experimental practice. This does not mean, however, that the account sketched here is necessarily confined to experimental practice. I do think that a similar story can be told about the way in which scientists have constructed models of particular target systems, one that focuses, as Massimi 2018b 2019b) does, more specifically on how scientists explore a target system's space of modality through modeling. In this paper, I focus on experimentation, however, because, while Giere (2006, p. 93) has suggested that the study of experimentation offers an excellent way to investigate the role played by perspectives in the production of scientific knowledge, they have not yet received the attention that, for example, models have received in the perspectivist literature ${ }^{1}$ will try to illustrate, through my discussion of perspectivism and experimentation, that Giere is correct about this, and that experimenters' regress situations in particular can offer us a good way to study the way in which perspectives emerge, transform and stabilize over time. As such, my hope is that this paper can give rise to more perspectivist investigations of experiments, to more philosophical attention for the production of knowledge claims, and to perspectivist studies of similarities and differences in the role played by models and experiments in knowledge production.

\footnotetext{
${ }^{1}$ Evidence for this claim can be found, for example, in the fact that in a recent edited volume on perspectivism Massimi \& McCoy 2019, there are no chapters explicitly concerned with experimentation, whereas many of them discuss models and representation.
} 


\section{Giere and Chakravartty on experimental detections}

The use of perspectives, as Chakravartty 2010 , p. 406) points out, derives from the fields of art and cartography ${ }^{2}$ They are used there for the representation of three-dimensional entities in a two-dimensional way. Perspectives allow for this because they have two significant characteristics. First, by idealizing and abstracting away certain aspects of the original, they actually offer the user the impression of viewing the original. And second, because their mathematical machinery allows for the variation of parameters, users can construct different perspectives on the original, each of which can have its own benefits and disadvantages. Because of these characteristics, the most saliant epistemological consequence of perspectives in these fields, according to Chakravartty, is that they "may yield different and apparently conflicting descriptions of their subject matter" 2010, p. 406).

According to Chakravartty, perspectivists now claim that this point also applies to science 3 Scientific instruments and theories, they claim, are to be seen as offering different perspectives on a particular target systems ${ }^{4}$ By means of a discussion of how scientists in astronomy $2006, \mathrm{p}$.

2 See (Giere 2006, p. 13) or van Fraassen 2008, p. 59) for similar discussions of the role of perspectives in these fields.

3 The perspectivist approach, according to Chakravartty, can be found in the work of Ron Giere (2006) and Paul Teller (2001). He also identifies some perspectivist ideas in Bas van Fraassen's (2008) work, but he does not take him to offer a full-blown perspectivist account.

4 A paradigmatic case of this claim for many perspectivists is offered by fluid dynamics, since it offers different, incompatible ways to model the behaviour of fluids. Which model one is supposed to use depends on the type of issue one is dealing with: if the goal is to explain, for example, how water flows or how waves propagate in a fluid, one will opt for the continuous, incompressible fluid model provided by the perspective of hydrodynamics; if, on the other hand, one wants to explain for example diffusion phenomena, the discrete particles model provided by the perspective of statistical mechanics will be preferable. This choice makes a difference, for as Massimi $2018 \mathrm{a}$ p. 350) points out, from the perspective of hydrodynamics, viscosity, for example, is a fundamental property of water, whereas it does not figure directly in the perspective of statistical mechanics. For different discussions of fluid dynamics as a case for perspectivism, see e.g. (Rueger 2005 Giere 2009, Morrison 2011 Rueger 2016 . 
$42-48)$ and neuroscience 2006 , p. $49-56)$ arrive at experimental detections, i.e. instrumentally produced representations of particular aspects of a target system, Giere has argued, for example, that the knowledge claims produced in these fields by means of such detections should be seen as perspectival. By this he means that we should not take them as representations of how the target system is like in itself, but rather only as representing what the target system is like from the perspective of the particular instrument used to produce the knowledge claim. The reason for this is that these instruments inherently mediate or influence the information we can obtain from these target systems:

Humans and various other electromagnetic detectors respond differently to different electromagnetic spectra. Moreover, humans and various other electromagnetic detectors may face the same spectrum of electromagnetic radiation and yet have different responses to it. In all cases, the response of any particular detector, including a human, is a function of both the character of the particular electromagnetic spectrum encountered and the character of the detector. Each detector views the electromagnetic world from its own perspective. Every observation is perspectival in this sense. (Giere, 2006, p. 48)

In itself, this claim can still be taken as merely offering a description of a fact about scientific practice: the use of different instruments leads us to different representations of the target system since, as Massimi (2012, p. 29) summarizes it, instruments are only responsive to a selected input range and the output they produce is inherently a result of the interaction between the instrument and the target system. According to Chakravartty, however, perspectivists then turn this characterization of an existing scientific reality into a philosophically substantive position by claiming that it entails that the only scientific knowledge we can have is of perspectival facts. Chakravartty characterizes such facts as follows: 
I will use the term 'facts' to denote true propositions, whatever analysis one may wish to give of the latter. A non-perspectival fact about a target system is thus a proposition that is true, independently of any particular perspective one may take with respect to it; it is true across perspectives. A perspectival fact is a proposition that is only true from, or within, or relative to, a given perspective (or limited set thereof). (Chakravartty, 2010, p. 407)

Chakravartty's reason for this claim is that perspectivists such as Giere (2006, p. 81) see "truth claims [as] always relative to a perspective." In the case of fluid dynamics (see footnote 4), for example, this would mean that there is no perspective-independent fact of the matter with regards to what water is: all we can say is that, from one perspective, water is an incompressible, continuous medium, while from another perspective, it is a collection of particles. Given that scientific practice inherently provides us with different perspectives, this perspective-relative view of truth entails, according to Chakravartty, that we end up with "the relativistic thesis that different perspectives inevitably yield irreducibly incompatible claims to knowledge" (2010, p. 407). Because of this, perspectivists cannot be realists, since realism comes down to the claim that our best science is capable of arriving at non-perspectival facts (Chakravartty, 2010, p. 406).

That the use of instruments often leads to multiple, incompatible and irreducible scientific representations of the same target system does not have to mean, however, that we can only know perspectival facts, according to Chakravartty. It can equally well be accounted for in dispositional terms. On such an account, the observable properties manifested by a target system depend on the specific circumstances in which the target system's causal properties are placed, and hence we can have different representations. But this is a consequence of the use of different experimental circumstances, not of the existence of perspectival facts: these different representations can be taken as "manifestations of one and the same property nonetheless" Chakravartty, 2010, p. 409). In this way, we can prevent the perspectival descent into relativism, since the experimental 
knowledge claims obtained are no longer to be read as stating that from the perspective of set-up $A$, the target system has property $x$ that makes it behave in way $\alpha$, and that from the perspective of set-up $B$, the target system has the property $y$ (incompatible with $x$ ), that brings the target system to act in way $\beta$. They are rather to be read as stating that it is a fact that the target system is endowed with a causal property $z$ that, given the experimental circumstances $A$, will dispose the target to behave in way $\alpha$, and given the circumstances $B$, will make the target behave in way $\beta$. While experimental instruments are required to produce such knowledge claims, their truth is not relative to the instruments' perspectives: "the facts produced by these investigations are perfectly non-perspectival, regarding the interactions of certain properties in specific circumstances" (Chakravartty, 2010, p. 409).

\section{Massimi's reply: perspectivism as historically situated realism}

In her (2012) response to Chakravartty, Massimi agrees with him that we should take science to be capable of providing knowledge of non-perspectival facts. And she even concurs that, at least in the case of experimentation, this knowledge can very well be understood in terms of what she calls his Dispositional Identity Thesis (DIT), which is the claim that "causal properties are identified by the dispositions they confer on objects" (Massimi, 2012, p. 31). However, even then, she claims, there is still room and actually a need for a philosophically substantive epistemic form of perspectivism, one which is concerned with "how we gain scientific knowledge of nature" (Massimi, 2012, p. 33). While our beliefs about nature are made true by non-perspectival facts, she will argue that our justification for them, i.e. our belief that they have been obtained by means of a reliable procedure, is perspectival in nature.

If pressed to account for how we gain scientific knowledge of nature, the dispositionalist will reply, according to Massimi (2012, p. 33), that it is through reliable measurement devices: 
these allow us to detect the behaviours that a target system is disposed to, and in this way, following DIT, they provide us with information about the system's causal nature. But the question can then in turn be raised how we gain knowledge of the reliability of such experimental procedures, i.e. how the beliefs about a target system's causal nature are in turn justified. To this, Massimi continues, the dispositionalist will reply that scientists obtain such justification by means of an inference to the best explanation: "believing in those causal properties is the best explanation for the success of our scientific instruments and detection procedures in delivering reliable beliefs" 2012 p. 34$)$. The success of a reliable experiment to provide causal knowledge thus also provides a scientist, according to the dispositionalist, with the belief that the experiment has indeed operated reliably. What is significant about this reply, according to Massimi $[2012$, p. 34 , footnote 18 ), is that it is reliabilist in nature: even though we often do not have an adequate causal understanding of how our experimental detection procedures work exactly, we can still take them to provide justified beliefs about the causal nature of the target system because their success shows that they are reliable procedures.

However, the problem with this reply, Massimi argues by means of work by Jonathan Vogel 2000, 2008) on reliabilism, is that it appeals to a bootstrapping mechanism of justification, i.e. "it sanctions its own legitimacy" (Massimi, 2012, p. 35). More specifically, the claim is that the dispositionalist's appeal to inference to the best explanation entails that if scientists obtain beliefs by means of reliable experiment, they immediately can also infer the belief that the experiment has operated reliably: in the case of J.J. Thomson's cathode ray experiments, for example, the dispositionalist's view would allow Thomson to infer from his reliably operating cathode ray experiments not only that these rays were made up of minuscule, negatively charged particles, but also that the electrometer employed in these experiments was reliable Massimi, 2012, p. 35 - 38). The problem with this is that it is not the way in which Thomson could have actually arrived at the belief that his cathode ray experiments functioned reliably. To obtain this belief, 
he would rather "have to test the electrometer, check that it is properly wired, that its meter reading is not faulty, calibrate it, and so on" Massimi, 2012, p. 38).

The bootstrapping problem arises, according to Massimi, because dispositionalism does not offer an independent way for scientists to obtain a justification for the beliefs about the causal properties of target systems that they infer from the dispositional behaviour observed in reliably operating experiments. To argue for this, she makes use of the distinction made by Ernest Sosa (1991) between apt and justified beliefs. An apt belief is one obtained in a reliable way, i.e. via "a way of arriving at belief that yields an appropriate preponderance of truth over error" Massimi, 2012 p. 43 , footnote 28). But in order to obtain the reflective belief that such an apt belief is also justified, i.e. to obtain the belief that it results from a way that yields a preponderance of truth over error, scientists cannot just appeal to the reliable experiment itself: having an apt belief is not sufficient to arrive at the belief that the apt belief is also justified. Such a reflective belief, which states that the belief about a target system's causal properties obtained via reliable experiments is justified because it is indeed obtained via reliable means, is rather arrived at by checking whether the belief about the causal nature of the target system coheres well with what Massimi calls the scientist's epistemic perspective. This perspective consists of beliefs that the scientist has about the target system, about the functioning of the set-up and her perceptual system and cognitive faculties, and about the reliability of these as sources of beliefs Massimi, 2012 p. $40-41$ ). What is important is that these beliefs that make up a scientist's perspective are independently acquired: Thomson obtained his beliefs about the reliability of his electrometer not from the results of the cathode ray experiments in which he used it, but rather from separate tests. In this way, perspectives offer scientists with an independent way to see that the beliefs about a target system's causal nature obtained by means of reliable experiments are indeed justified: 
By occupying an epistemic perspective, the agent is able to self-reflect on her beliefs, on the sources of her beliefs, the way beliefs cohere with one another, no less than the way in which they, individually and jointly, are anchored to the empirical ground via reliable methods. (Massimi, 2012, p. 49)

This justification is perspectival, since depending on the other beliefs that make up a particular scientist's perspective, the justification she will provide will be different: insights obtained in response to later developments can entail, for example, that the scientist has to give up certain beliefs belonging to her perspective, which will lead her to rethink whether, and if so, how, the belief about the target system's causal properties coheres with what she now beliefs about the reliability of the experiment. In Thomson's case, for example, his epistemic perspective brought him to believe that his experiments provided reliable evidence not just for the claim that cathode rays consist of negatively charged particles, but also for the claim that electrons were structural features of the ether. Later theoretical and experimental investigations showed him, however, that the sources from which he derived this belief about ether-structures were not reliable, and hence it was no longer coherent with his epistemic perspective, which made him give it up (Massimi, 2012 , p. $42-48)$.

This perspectival view of belief justification does not lead to relativism of the sort that Chakravartty warns for, however, since it does not affect whether or not the experiment from which the belief about the target system's causal properties is derived, is actually reliable. Even though Thomson, given his perspective at the time of his experiments, was justified in believing that electrons were structural features of the ether, this perspectivally obtained justification does not determine whether or not electrons are actually of such a nature. That is rather, as Massimi puts it, a perspective-independent fact of the matter: 
[W]hether something is a reliable source of truth or true belief is not context-dependent or perspective-dependent. There are facts of the matter that make our beliefs about nature either true or false, and these facts of the matter are not perspectival or context-dependent. Reliable methods and procedures ultimately tell us whether or not the electrons really have the mass-to-charge ratio that Thomson found, and whether or not electrons are structural features of an elastic ether. (Massimi, 2012, p. $48-49$ )

\section{Perspectivism and the epistemological dynamics of experimentation}

Giere's perspectivism, we have seen in section 2, was concerned with the way in which instruments provided scientists with perspectives that allowed them to produce experimental detections of a particular target system. Chakravartty argued, however, that in combination with Giere's claim that truth is relative to a perspective, the fact that we will always have incompatible perspectives entails that we end up with the relativistic thesis that science can only provide irreducibly incompatible truths. To prevent this, Massimi then argued that perspectives only play a role in the justification of experimental knowledge claims, i.e. in the evaluation of an experimental instrument's reliability. Whether or not such an instrument has in fact produced a true experimental knowledge claim is, on the other hand, a perspective-independent matter: perspectives do not play a role, on her view, in the construction of a reliable experiment. As such, perspectivism can no longer say anything about the production of knowledge claims: whether experimental instruments are reliable, and hence lead to true knowledge claims, is a perspectiveindependent fact of the matter, and that's that.

In what follows, I will argue, however, that we can allow for perspectives to paly a role in the production of experimental knowledge claims as well, without ending up with relativism. My starting point for this will be Giere's own claims about how scientists handle incompatibilities 
between different perspectives, since what threatens to render Giere's perspectivism relativistic, according to Chakravartty and Massimi, is that it does not allow scientists to overcome such incompatibilities: perspectives, on Chakravartty's presentation of Giere's perspectivism, are irreducibly incompatible, and in combination with Giere's claim that truth is always relative to a perspective, this entails that we end up with irreducibly incompatible truths $5^{5} \mathrm{My}$ starting point for this will be the following claim by Giere, made in a section on the compatibility of instrumental perspectives, concerning situations in which scientists are confronted with instruments that produce incompatible representations of a target system:

$[\mathrm{W}] \mathrm{e}$ expect different visual systems viewing the same scene from the same location to be compatible because we operate with the methodological presumption that the total electromagnetic field is unique. Different images produced must be due to differences in the makeup of the different visual systems. There is nothing at all contradictory about different systems producing different images with the same input.

This is not to say that different instruments might not yield results that appear to conflict. Two different gamma ray detectors may overlap in the range of energies of gamma rays to which they are sensitive. So it is possible, for example, that one instrument might indicate that, over a given period of time, there is a considerable flux of gamma rays of energy 10 $\mathrm{MeV}$ coming from a well-defined source, while the other indicates hardly any flux at that energy during that period of time. The relevant group of scientists confronted with this

\footnotetext{
5 That Massimi also ascribes to this characterization of Giere's perspectivism can be seen from her claim that she developed her perspectivism in response to the worries with Giere's perspectivism that "there is no specific way the observed objects are in and of themselves, independently of the particular perspective from which they are observed or detected" (2012, p. 29) and that "as soon as the prefix "From where we stand ..." is added, and truth claims are made relative to a perspective, the ghost of relativism comes back to haunt the perspectivist" (2012, p. 30). See also Massimi's (2015) discussion of how Giere's perspectivism and his reading of Kuhn on which it is based can lead to relativism with respect to both natural kinds and truth.
} 
situation would draw the conclusion that one or the other instrument is malfunctioning and proceed to try to figure out what had gone wrong. They would not accept the result as simply a curiosity of nature. This behavior would be in accord with the methodological principle of proceeding as though nature has a unique causal structure. Of course, one does not expect working scientists explicitly to invoke anything so grandiose. That is just how I recommend we understand their activities. (Giere, 2006, p. 57)

This quote shows that Giere distinguishes unproblematic incompatibilities from problematic ones. If different instruments produce incompatible representations, they are unproblematic if the scientists know that the incompatibility results because the instruments interact in different ways with the target system. They are problematic, on the other hand, if, given the scientists' beliefs about how the instruments are supposed to function, they did not expect such incompatibilities to arise. This they take to indicate that one of the instruments has not operated reliably, and they will hence turn to an investigation of the instruments and their functioning in question.

Giere does not really expand on this idea further, which is unfortunate, since it concerns an issue about which Massimi cannot really say anything more either. Her perspectivism only concerns how scientists come to recognize that an experiment is operating reliably: as she puts it in her (2018a, p. 343, footnote 2; original emphasis) characterization of perspectives, one of their elements are "the experimental, theoretical, and technological resources available to the scientific community at the time to reliably make [...] scientific knowledge claims". As such, it does not concern cases where it is unclear or disputed whether the experimental resources availbel to scientists allow them to reliably make knowledge claims or not.

The reason for restricting perspectivism to cases where we are dealing with reliable instruments, it semes, is to ensure tha tit can also form a realist position: if perspectives played a role not only in the justification of experiments that are in fact reliable, but also in in the production of experiments about which it is uncertain or disputed whether they are reliable, then it would 
seem that we have no way to guarantee that perspectives allow scientists to track perspectiveindependent truth, as Massimi (2018a) takes to be their role if we want perspectivism to allow for realism.

In what follows, I will argue that perspectivism can be extended to cover also the production of experimental knowledge claims. I will do this by elaborating further Giere's suggestion about how scientists deal with problematic incompatibilities. My starting point for this will be Uljana Feest's (2016) work on experimenters' regress arguments, which are situations in which scientists are confronted with incompatible experimental detections and uncertainty about the reliability of the experiments that produced them. Such situations can arise, according to Feest, when it is actually indeterminate whether an experiment is reliable or not. As such, the discussion that is to follow differs from the experiments discussed by Massimi $(2012,2019 a)$, where we have good grounds to believe that we are in fact dealing with reliable experiments.

While Feest does not formulate her analysis of how scientists handle such situations in perspectivist terms, I will argue that it can provide us with a better understanding of the role played byu perspectives in the way in which scientists transform an experiment about which it is indeterminate whether it is reliable to a reliably operating experiment. This will bring me to reconceptualize perspectives as embodied and situated ways of going about in experimental practice which can become explicated through a process of interpretation. While this entails that perspectives no longer function as truth-trackers, as they are on Massimi's view, but rather as ways to explore how a reliable experiment could possibly be constructed, I will then argue that the view presented here still allows for realism. 
4.1 Feest on experimenters' regress arguments

An experimenters' regress situation arises when scientists are confronted with an experiment $B$ that was carried out to replicate an earlier experiment $A$, but produced different results. In such a case, the scientists involved may disagree about whether $B$ should be taken as a replication of $A$ : those who conducted $B$ may argue that, because they take this experiment to have operated reliably, it is a replication and it refutes the results of $A$, while those who conducted $A$ may argue that $B$ is not a replication, since its results are not in line with the results they obtained, and hence it should not be taken as reliable (Feest, 2016, p. 35). As such, regress situations present exactly the kinds of situations that are at issue in how Giere's perspectivism deals with problematic incompatibilies: if $A$ and $B$ are supposed to be replications, but they produce incompatible results, can scientists overcome such situations, and if so, how?

This question is also the central philosophical issue in the debate on experimenters' regress situations between Harry Collins and Allan Franklin (see (Feest, 2016, p. 35 - 26) for references). According to Collins, such situations cannot be overcome in a rational, objective way, because in their judgments about the reliability of an experimental set-up or about the validity of experimental results, scientists inherently rely, in part, on experimental ability. This is a certain experience in going about in experimental practice, which is obtained and developed through practice. Because of this, it can never be fully explicated or established ${ }^{6}$ Hence, when scientists try to justify their judgments in the case of a disagreement, they "will each ultimately appeal to a 'gut feeling' that cannot be further justified, thereby making it impossible to decide on rational grounds whether one experiment $B[\ldots]$ is in fact a precise replication of another experiment $A$ " (Feest, 2016, p. 37).

${ }^{6}$ As Feest 2016 p. 37) points out, Collins elaborates his notion of experimental ability on the basis of Ludwig Wittgenstein's work on rule-following. For overviews of the literature on ability, know how and tacitness, see Rouse 1987 p. 100, footnote 59), (Soler.2011 p. 397 - 399) and (Turner 2014 chapters 4-5). 
According to Franklin, however, the influence of such experimental ability is confined to the actual production of the experimental results, what he calls 'getting an experiment to work'. It does not present an epistemological problem, he argues, for when scientists turn from the production of experimental results to their justification, what Franklin calls 'demonstrating that it is working properly', they rely on explicit epistemological strategies which they know to be justified $!^{7}$ As such, Franklin agrees with Collins that scientists can disagree in how they actually get an experiment to work reliably, because they operate with different experimental abilities. Still, by explicitly following these strategies, they can arrive at an objective agreement about whether the experiments have reliably produced valid results, and hence about whether they count as replications. As such, scientists do have at their disposal ways to rationally decide regress situations (Feest, 2016, p. $35-36)$.

By means of a study of an experimenters' regress situation in the cognitive sciences, Feest argues that neither Collins nor Franklin presents a completely adequate epistemological analysis of such situations. Central to her argument is the claim that to construct experiments that could provide information about the questions under investigation, scientists first have to operationalize the concepts that figure in those questions, which means that they have to decide "what kinds of experimental data to treat as indicative of the subject matter at hand" (Feest, 2016, p. 36). Feest's case concerns experimental investigations of the Mozart effect, i.e. a positive influence of Mozart Music on spatial reasoning (Feest, 2016, p. 38 - 40). In the original experiments, the possible effect of Mozart music on spatial reasoning was operationalized in terms of performance on three spatial reasoning tasks of the Stanford-Binet intelligence test. Given that the experimental

\footnotetext{
7 Feest gives the following list of examples of such strategies: "(1) appeals to a well-corroborated theory of the apparatus, (2) the use of different experimental apparatuses, (3) the demonstration that the same apparatus can detect similar phenomena, (4) the test of predictions about the results of an experimental intervention, and many others (the list is open-ended)" (2016 p. 35).
} 
participants who were subjected to Mozart's Sonata for two Pianos in D-major performed better on these tasks than those who were subjected to relaxing music or silence, it was claimed that these experiments indicated that there was such a thing as the Mozart effect (Feest, 2016, p. 38)

This experimental inference from the data produced in the experiments to the claim that there is such a thing as the Mozart effect was soon criticized, however. This was done by explicating and scrutinizing experimentally some of the assumptions underlying this inference. One criticism was that the experiments could not be taken as reliably showing the existence of a Mozart effect, but rather only of a mood effect, since the effect could equally well be brought about by upbeat music that put the participants in a good mood (Feest, 2016, p. 41). Others disputed that the experiments could be reliably taken to show the existence of any kind of effect, since if spatial reasoning was measured by means of different tests, it was "impossible to replicate" (Feest, 2016. p. 39).

The scientists who had carried out the original experiments, however, did not take these criticisms to show that there was no Mozart effect. They rather took them to mean that the effect they had discovered was more narrow, claiming that "there are different categories of spatial abilities (spatio-temporal processes vs. spatial recognition) and that [they were] only claiming a Mozart effect on the former" (Feest, 2016, p. 39). The failure to replicate, they claimed, arose not because there is no Mozart effect, but rather because in the replicating experiments the relevant confounding factors were not controlled for: if the Mozart effect only concerns spatiotemporal processes but not spatial recognition, then experimental operationalizations that do not distinguish between the two will of course not display the effect in the way found in the original experiments. To this, critics in turn replied that the original experimentators were arguing for the existence of an effect that, it seemed, could only be brought about in the lab in which it was originally investigated (Feest, 2016, p. 39). Most scientists concluded from this that "in its most general formulation, the Mozart effect (as an effect of Mozart music on spatial reasoning) may 
be regarded as refuted" (Feest, 2016, p. 40). At the same time, however, it was not necessarily taken to mean that the original experiments were concerned with nothing at all: they did give rise to further, still ongoing investigations of the possible impact of music in general on different forms of intelligence (Feest, 2016, p. 38).

As such, at a certain point in the case, there was a clear experimenters' regress: the scientists involved disagreed about which experiments could be taken as reliable, and which could therefore be taken as providing information about the Mozart effect. The reason for this, according to Feest (2016, p. 40), was that there was a genuine epistemic uncertainty with regards to how this possible effect was to be operationalized, and whether the proposed operationalizations adequately implemented these concepts. Such uncertainties arise, Feest then argues, because the boundaries of the concepts figuring in the question under investigation are actually indeterminate:

This uncertainty $[\ldots]$ arises from the fact that scientists often use terms that are (at least for the time being) referentially indeterminate in the sense that they cannot yet be sure (a) whether the phenomena or entities in question really exist, (b) whether their operational definitions adequately individuate them, and (c) whether specific experiments adequately implement the operational definition (where this includes the question of whether all relevant confounding variables have been controlled for). (Feest, 2016, p. 38)

In the Mozart effect case, for example, there is no established agreement on what kind of spatial reasoning the different tests used measure exactly: "[the relevant subtests of the Stanford-Binet] are not linked to some well-confirmed theory of the cognitive and/or neural mechanisms that give rise to the behavioral responses measured by the test [since] no such theory exists" Feest, 2016, p. 40). The reasons for this, as Feest (2016, p. 40) points out, is that these tests are not validated by showing how they track a well-delineated neural/cognitive mechanism, but rather by showing that they correlate well with other tests taken to measure the same form of intelligence. As 
such, genuine disagreements can arise about which specific experiments are reliable in measuring spatial reasoning, since it is indeterminate how to operationalize the concept 'spatial reasoning' adequately.

Feest then argues that contrary to what Franklin assumes, such regress situations cannot be overcome by means of epistemological strategies (see footnote 7). To carry out these strategies, scientists will equally well have to choose how to operationalize spatial reasoning, and given that it is indeterminate and disputed how to do so correctly, they will equally well disagree about whether these strategies have been carried out in a reliable way. Hence, if there is indeterminacy, there is no objective way to decide which experiments can be taken to provide reliable information about the question under investigation (Feest, 2016, p. $37-38)$.

As such, Feest takes her case study to validate Collins' analysis, but only up to a point. Collins is correct, according to Feest (2016, p. 37), in claiming that ability, in the form of tacit aspects such as skills, materials and background assumptions, plays an important role in the way in which scientists, both in the production and evaluation of experimental knowledge claims, operationalize the concepts figuring in the question under investigation. And in cases where it is indeterminate how to operationalize adequately, there is no way to fully explicate those tacit aspects and distinguish the relevant and confounding factors from the non-significant ones, since this distinction is indeterminate as well.

Contra Collins, however, Feest does not take this to mean that no progress can be made in such situations. In the Mozart effect case, for example, such progress can be found in the dialogue between experimentators and critics, which offers "a gradual process of explicating and testing the hidden premises that are required for specific experimental inferences (and thus, [...] a gradual refinement of central concepts at stake)" (2016, p. 43). This process that scientists engage in when dealing with regress situations Feest (2016, p. 39) calls 'operational analysis'. When scientists are confronted with a regress situation, they will try to make explicit those tacit aspects they 
take to underly the experimental operationalization they see as problematic, and then investigate theoretically and experimentally whether these assumptions prove robust. Even though, given the indeterminacy of how to operationalize the question under investigation, we cannot expect such operational analyses to decisively adjudicate regress situations in a completely objective way, scientists in this way can still arrive at a better understanding of how to operationalize.

That regress situations cannot be resolved in a completely rational way is not a problem, however, or at least not a problem concerning how scientists handle regress cases, according to Feest. It is rather a problem concerning what philosophers expect from experiments. On her view, the idea that regress situations need to be resolved in a fully decisive and objective way stems from a rather narrow philosophical conception of the role of experiments in science. On this view, experiments are primarily concerned with objectively establishing the validity of particular knowledge claims: experiments need to decisively establish whether or not there is such a thing as the Mozart effect. Both Franklin and Collins take this view for granted: Franklin believes that they can do this, and hence he sees regress situations as rationally decidable, while Collins "even in denying that experiments can provide decisive answers between different hypotheses, still holds on to the idea that this is their primary job" (Feest, 2016, p. 41). Given the indeterminacy underlying regress situations, however, experiments in such cases cannot be expected to provide such answers, according to Feest. What they can do, however, is help scientists in articulating a better understanding of how the questions under investigation can be operationalized. On this view, experiments are exploratory, in the sense that they offer scientists a way to explicate and investigate further the tacit assumptions underlying particular operationalizations, and hence a way to make progress with respect to the epistemic uncertainty that characterizes their field of research (Feest, 2016, p. 41) ${ }^{8}$ Therefore, Feest concludes, her discussion should equally well be

\footnotetext{
8 See Steinle 1997 Feest 2003 Schickore 2016 Steinle 2016 for some discussions of exploratory experimen-
} tation and its history as a philosophical concept. 
read as a call for philosophers to "turn our attention away from hypothesis testing (as traditionally conceived), and instead take into view the dynamic process by which experimental scientists generate knowledge by constantly revising and adjusting their central concepts" (2016, p. 43).

4.2 Perspectives and the process of interpretation

At the end of section 3. I claimed that Massimi's perspectivism cannot say much about situations where scientists disagree about the reliability of an experiment, since her perspectivism is concerned solely with how scientists come to see that an experiment is reliable, in cases where the experiment is indeed reliable. In terms of Feest's analysis, her concern is solely with situations in which scientists have at their disposal a determinate way to operationalize the questions under investigation.

Regress situations are different, since there, according to Feest, it is indeterminate how to operationalize. Such situations, it seems, are very similar to the ones I have described, following Giere's claims about the compatibility of instrumental perspectives, as involving problematic incompatibilities. On this reading, when scientists are confronted unexpectedly with one detector indicating a significant flux of gamma rays and another one indicating no flux, we can say that at least one detector relies on a problematic operationalization of the questions that the detectors are supposed to provide information about (see the quote on page 13). In what follows, I will try to further elaborate Giere's suggestion of how scientists can address such problematic incompatibilities by reflecting on what Feest's notion of operational analysis could mean for perspectivism. This will lead me to argue that if we reconceptualize the notion of perspectives in a way that focuses more on the tacit and exploratory aspects of experimentation, we can extend Massimi's perspectivism in such a way that it covers both the production and justification of experimental knowledge claims, while still allowing for realism. 
What this issue shows is that Massimi's perspectivist account of justification falls prey to the same issue as Feest raises for Franklin $9^{9}$ And the reason for this, it seems, is that Massimi works with the same hypothesis-testing epistemology of experimentation as Franklin: on her view as well, it is a fact of the matter whether or not an experiment is reliable, and if it is reliable, it can be taken to provide a perspective-independent ground for the truth or falsity of experimental knowledge claims (see the quote on page 11). In line with Feest's claim that the issues that Franklin's account has with regress situations can be overcome by replacing this epistemology with one that pays more attention to the exploratory aspects of experimentation, I will argue that the issue with Massimi's perspectivism can be addressed if we pay more attention to the role played by perspectives in the production of reliable experiments, rather than solely focusing on how they allow scientists to justify experimental knowledge claims. To do this, we first need to elaborate further Giere's suggestion about how scientists address the issue of incompatible perspectives (see the quote on page 13). I will do this by means of a discussion of what Feest's notion of operational analysis can teach us about perspectives.

On Feest's account, when confronted with a regress situation, scientists will try to explicate and scrutinize the tacit elements involved in the operationalization that led to the production of a disputed experiment. In perspectivist terms, we could say that they engage in a process of interpretation, in which they try to make sense of the perspective that guided the scientists who carried out the experiment. This is done by ascribing particular beliefs and actions to these experimentators, beliefs the evaluators themselves hold about the target system or the functioning of the set-up, and actions that the evaluators themselves would have undertaken were they to carry out an experiment of the kind under scrutiny. In this way, they try to reconstruct the

\footnotetext{
9 The similarity between Massimi's position and Franklin's shows itself in that Franklin's epistemological strategies (see footnote 7) offer scientists ways to obtain the beliefs that, according to Massimi, make up their epistemic perspective (see page 9 .
} 
inference that could have led the experimentators from the production of the experimental data to the formulation and validation of the experimental knowledge claim under scrutiny. When this does not work out completely, they will start tinkering with the elements of the inference, replacing them by beliefs and activities that they do not themselves hold or would carry out, but which they take to be plausibly ascribable to the experimentators. At the same time, they will also start testing this inference and the elements they take to be part of it, by trying to experimentally replicate it, by consulting articles or earlier experiments on the subject of the knowledge claim under investigation, and, if possible, by engaging in dialogue with the scientists whose actions they are trying to understand, and asking them questions about how the experiment was carried out exactly 10

Given that this process of interpretation is concerned, at least in part, with making explicit the tacit elements underlying the experimental inference, it could be that the scientists whose actions and reasoning are being interpreted do not recognize the beliefs or actions ascribed to them, or proclaim that they did not have commitments to any specific beliefs when carrying out the experiment. For all we know, this could be true, and in that case it will lead to a public dialogue between experimentators and evaluators. In any case, to understand what the experimentators were doing, some kind of public account of how one could arrive at their conclusion is needed, since only on such a basis can the experimental knowledge claims about which there is a disagreement be scrutinized. As such, on the view presented here, perspectives can be seen as a collection of actions and beliefs that are publicly ascribed to experimentators, in order to make sense of how they went about in their experimental work 11

10 See Soler 2011 for a very extensive discussion of the different ways in which scientists can engage with the work of others in order to arrive at a better understanding of the possible tacit aspects that go into the production and validation of this work.

11 This conceptualization of perspectives as constructed through a process of dialogue and interpretation comes quite close to an idea recently formulated by Massimi, which is that "[t]he willingness to engage with other 
While such public interpretations are produced in cases of explicit disagreement, this does not mean that scientists only engage in interpretation in such cases. Most of the time, interpretation is just not an activity they carry out explicitly besides all the other activities that make up their experimental practice. In fact, on the view presented here, whatever scientists do in experimental practice can be seen as an interpretative activity ${ }^{12}$ everything they do, from constructing a set-up over accounting for data to trying to replicate an earlier result, is done in response to what other scientists have done, and hence it involves trying to make sense of the perspective that guided these other scientists. Most of the times, however, these interpretations are not made explicit, because scientists normally have a fairly good intuitive grasp of why other scientists do what they do. The tacit then remains tacit, and hence in such cases we can understand perspectives as embodied and situated ways of going about in experimental practice. I take perspectives to be embodied in the sense that they concern the bodily experimental abilities that scientists have, where bodily is taken in the broad sense as also concerning cognitive abilities. I take them to be situated in the sense that this going about is not only influenced by the bodily abilities that scientists have: how they go about is equally well influenced by the specific material and cultural setting in which they operate.

If perspectives are primarily embodied and situated ways of going about in experimental practice, this entails that scientists will normally not even have an explicit understanding of their own perspective. This is not as problematic as it sounds: as has been pointed out in the epistemic agents occupying different scientific perspectives (synchronically and diachronically) is [...] key to perspectivism as a pluralist view about ways of knowing" (2019a p. 11). (At the time of writing, this article (Massimi, 2019a) is online-only. Hence, page-references are not to the journal page numbers, but rather to the page numbers of the pdf-version found online.) The conceptualization as it is presented here is based on Feest's account of tacit knowledge as "something that is only constructed in the process of "explication," where such explications can be tailored to specific purposes" (2016 p. 42). Feest in turn acknowledges that her view derives from Stephen Turner's (2014) work on tacit knowledge.

12 This is an idea I take from Joseph Rouse's (1987) practical-hermeneutical account of experimentation. 
literature on tacitness and know how (see footnote 6 for overviews of this literature), it is very difficult to give an explicit account of one's experimental abilities, i.e. of why one carries out an experimental activity in the way one does. The reason for this is that one learns how to carry out such activities not by consulting an explicit list of instructions, but rather by seeing how it is done, and by trying it out yourself. As Hacking puts it: "you learn to see through a microscope by doing, not just by looking" (1983, p. 189) and "[o]ne needs theory to make a microscope. You do not need theory to use one" 1983 p. 191). While we can thus say that scientists' experimental activities are guided by a certain perspective, this does not entail that these scientists have a fully explicit understanding of how they go about, and why they do it as they do.

It may seem that we now end up with two different notions of perspectives: one that conceptualizes them as tacit and embodied ways of going about in experimental practice, and one that takes them to be the beliefs and actions that other scientists publicly ascribe to scientists who have carried out experiments. These two notions are, however, two sides of the same coin, and they are linked through the process of interpretation: when scientists disagree or have issues with an experimental knowledge claim formulated by others, they will try, through interpretation, to construct a public account of the embodied and situated way in which the experimentators went about, in order to be able scrutinize and test it. In this way, we come to see why experimenters' regress situations offer a very suitable way to study perspectives: such situations exactly bring about this demand for explicit interpretation, and as such, they offer us a way to study how scientists conceptualize the way in which they themselves as well as others normally go about in experimental practice, and how the regress situation is different.

The process of interpretation should moreover not be seen as merely providing understanding of how other scientists went about in the production of an experimental knowledge claim. It is, at the same time, also an exploration of our own perspective. By ascribing to others actions we would undertake and beliefs we hold to be true, and by then tinkering with them in order to make 
sense of the experimental inference, we at the same time acquire insight into our own perspective, and in how it differs from that of others. This can then also entail that, by scrutinizing and testing the beliefs and skills we ascribe to others, we change our understanding of our own perspective as well. As such, interpretation is not a process that operates in just one direction: given that it involves, in part, dialogue with those whose experimental inference we want to understand, either through direct communication or by engaging with their published work, it is rather a process that transforms the perspectives of both those being interpreted and those who do the interpreting.

In this way, we come to see how the process of interpretation sketched here is in line with Feest's call to pay more attention to "the dynamic processes by which experimental scientists generate knowledge by constantly revising and adjusting their central concepts" (2016, p. 43). Successful interpretation, which means that both the experimentators and the interpreters agree on the plausibility of the explicated perspective, does not establish the perspective as offering the correct way to operationalize the questions under investigation. Rather, it should only be seen as stabilizing a perspective that offers a possible way to operationalize. This does not mean that there are no other ways to operationalize, and given that there will still be tacit elements underlying the operationalization, this perspective is still open for further interpretation. As such, perspectives are historically dynamical entities: they can emerge through the process of interpretation, but this process can equally well transform them, and given that the list of tacit elements is open-ended, this process of interpretation can never be settled once and for all. Hence, perspectives are to be seen as entities that are subject to constant historical change 13

Let me summarize what has been said above. Scientists working within a particular field can be taken to have a particular perspective on it, which is an embodied and situated way of

\footnotetext{
13 My inspiration for this conceptualization of operationalizations as stabilized rather than as established derives from Feest's (2011) work on the stabilization of experimental phenomena.
} 
experimentally approaching different questions regarding that field. When they set out to experimentally investigate a particular question, their perspective will bring them to operationalize the concepts that figure in the question in a particular way. Other scientists will then try, by engaging in interpretation, to construct an understanding of how the experimentators went about: they will try to explicate the activities and beliefs underlying the operationalization, in order to be able to scrutinize them. Through this process, they can arrive at a better understanding of how to operationalize the question under investigation, and hence they achieve an understanding not only of the perspective that guided the experimentators, but also of their own. In this way, the process of interpretation not only brings about perspectives in an explicit form; it at the same time also transforms them. And if the interpretation is recognized as plausible by all involved, we can say that a perspective, offering a possible, and partially tacit, way to operationalize the question under investigation, has been stabilized. This stabilization then invites scientists to explore the perspective through further interpretation.

\subsection{Perspectives, reliability and realism}

Massimi's perspectivism allows for realism because it takes the reliability of an experiment to be a perspective-independent fact of the matter (see the juote on page 11). Perspectives, on this view, do not play a role in the production of experimental knowledge claims, and hence they cannot influence their truth. They are rather only concerned, as Massimi 2018a p. 343) puts it, with "tracking [the] perspective-independent states of affairs" that reliable experiments provide information about. As such, it is perspectives themselves that offer scientists a way to evaluate the reliability of an experiment, and hence a scientist can obtain a justification for an experimental knowledge claim from her own perspective. Perspectives can do this, because on her view, they are explicit sets of reflective epistemic beliefs and methods to which scientists have direct access. 
That they are explicit in this way is clear from the way in which Massimi characterizes them as offering scientists a way to self-reflect on their position as epistemic agents (see the quote on page 10, and also her more elaborate characterization of perspectives in (Massimi, 2018a, p. 343, footnote 2)).

On the view presented here, however, perspectives are not always explicit in this way: as pointed out in section 4.2 scientists themselves do not necessarily have an explicit understanding of the perspective that guides them in their experimental practice. This entails that a scientist's perspective in itself can not show her that the experiments she carried out are reliable, and hence, it can not show her that her experimental knowledge claims are justified: since it is not a set of explicit beliefs, a scientist cannot consult it in order to see whether an experimental knowledge claim coheres with it. This raises the question how scientists can come to recognize the reliability of an experiment. And given that on Massimi's account, it is this perspective-independence of an experiment's reliability that allows for realism, the claim made here that embodied and situated perspectives also play a role in the production of experimental knowledge claims equally well raises the question whether we can still be realists on the account presented here. It is these questions that I will address in what follows.

On the view presented here, the role played by perspectives in experimental practice is not primarily to establish an experiment's reliability. It is rather exploratory: they guide a scientist in her search for an operationalization that could lead her to the production of an experiment that can be taken as reliable. That she has achieved such an experiment is not something that she can recognize from her own perspective: it rather essentially involves that it is recognized as such by others from within their own perspective, which can occur when these others are able to understand and reproduce in their own way what was achieved in the original experiment. Only then can it be claimed that the original experiments operated reliably, and that their results can be taken to provide information about the target system under investigation. As such, reflective 
epistemic beliefs regarding reliability and justification can only emerge through the process of interpretation, which inherently involves dialogue with and scrutiny by others.

As such, that an experiment can be taken to be reliable, and hence to provide us with some knowledge about the target system, is the result of a whole experimental process. This process necessarily involves earlier experimental explorations of how to operationalize the questions we have regarding the target system, and later experimental replications of the experiment that is now recognized as reliable. Perspectives contribute to this process by guiding scientists in their search, through the process of interpretation, for an operationalization that is recognized by different scientists as adequate. On this view, the epistemological function of an experiment in scientific practice is thus not primarily that it offers scientists with a perspective-independent ground for truth or falsity. It can acquire such a function, but this can only result out of a whole process in which experiments primarily function in an exploratory way. As such, the primary epistemological function of experiment is that it contributes to the search for an operationalization of a question under investigation that enables scientists to construct experiments that offer the possibility of ascribing truth or falsity to particular experimental knowledge claims ${ }^{14}$

When scientists are able to construct an experiment that is seen as reliable in the way described above, they can take this to indicate that the operationalization used offers them a way to have their experimental practice latch on to reality in some sense. It means, more specifically, that they have found a way to experimentally intervene on the target system in such a way that in a particular experimental set-up, it can be reliably expected to display regular behaviour. In this way, we come to see how perspectives can contribute to the production of experimental knowledge

14 The view presented here is thus in line with Hacking's 1982 1991) conceptualization of experimentation as a particular style of reasoning, according to which the fact that we are in a position to ascribe truth values to experimental knowledge claims is the result of a contingent historical process in which the laboratory is developed as a space for experimental investigations and reasoning. See especially (Hacking 1992, p. 51, footnote 2) for how he sees the relationship between the laboratory and experimentation as a style of reasoning. 
claims: they guide scientists in their search for an adequate operationalization of the concepts that figure in the question under investigation. Moreover, through the process of interpretation, perspectives can also contribute to the justification of such claims, since this process, as was outlined above, brings scientists to explicate and replicate experimental knowledge claims, which can then inform them about whether or not the experiment on which these claims are based can be seen as reliable.

In this way, we can come to see how realism fits within the perspectivism presented here. Through the process of experimentation as it is sketched here, which inherently involves interpretation and replication, scientists can come to agree on which experiments have operated reliably, and hence on which experimental knowledge claims can be taken as true and which as false. This does not mean that they agree exactly on all aspects of how these knowledge claims are to be interpreted, since, for all we know, the operationalizations with which these scientists are working rely on different tacit assumptions. But given that this disagreement is implicit, if all scientists involved take the experiment to have functioned reliably, this implicit disagreement will not prevent them from ascribing the same truth values to the experimental knowledge claims at issue. The perspectivism presented here thus allows for the claim that reliable experiments can provide us with true experimental knowledge claims ${ }^{15}$

Moreover, given that, on the view presented here, perspectives concern not only the conceptual but also the practical and material aspects of how scientists operationalize research questions, we can equally well take it to allow for the claim that reliable experiments are concerned with mindindependent entities out there in the world. In a reliable experiment, scientists can be taken, more specifically, to be intervening on particular entities in such a way that they bring about stable empirical regularities in the experimental set-up ${ }^{16}$ This does not mean that they agree

\footnotetext{
15 I would like to thank two anynonymous reviewers for pushing me on these points.

16 Massimi (2019a) also indicates that she sees her perspectivism as compatible with James Bogen and James Woodward's (1988) interventionist view.
} 
exactly on every possible aspect of how the experiments are carried out, since for all we know, the operationalizations with which these scientists are working rely on different implicit skills. But given that this disagreement is implicit, and that the experiments are indeed recognized, through replications that vary on the set-up, as bringing about a stable and robust empirical regularity, this implicit disagreement will not entail that they are in fact manipulating different entities or that they have brought about an effect confined to a specific

The realism presented here is, of course, dependent on scientists finding operationalizations that allow them to construct experiments that are seen as reliable in the sense discussed here, i.e. as resulting out of a whole process of experimentation. I do not take this to be a problem, however, since given that the recognition of reliability necessarily involves replication by other scientists, the realism presented here is not perspective-relative. That the realism presented here depends on scientists finding an adequate operationalization rather solely indicates that it takes a lot of conceptual and practical work to get an experiment to work reliably. Once we get an experiment that is recognized as working reliably, however, there seems to be no reason why we could not be realists with respect to the knowledge claims and the empirical regularities that it is recognized as bringing about.

In this way, the perspectivism presented here is more encompassing with respect to experimental practice than Massimi's, since it covers not only the evaluation of an experiment's reliability, but also the way in which such a reliable experiment is produced. It emphasizes, more specifically, the amount of practical and conceptual work that is required to get an experiment to work reliably: it involves bringing together materials, skills, and beliefs in an experimental set-up in such a way that when others interpret and experimentally and theoretically scrutinize the functioning of the set-up, they come to see that it is indeed working adequately. And if we then follow the way in which such reliably working experiments are picked up and used by others in new experimental investigations, or how they are disputed, scrutinized and tested in experimenters' 
regress situations, we can come to see how, over time, perspectives can emerge, transform, and stabilize. In this way, it does indeed seem to be the case, as Giere $(2006$, p. 93) suggests, that experiments allow us to study the meshing of different perspectives.

\section{Concluding remarks}

In this paper, I have tried to elaborate an account of how we can understand the role played by perspectives in the production and validation of experimental knowledge claims. My starting point for this was Chakravartty's claim that Giere's perspectivism with respect to the role played by instruments in the production of experimental knowledge claims leads to relativism, since the presence of irreducibly incompatible perspectives entails, on this view, that we end up with irreducibly incompatible truths. In response to this, Massimi argued that there is still room, and actually a need, for perspectivism, but this only with regards to how scientists justify experimental knowledge claims: perspectives, on this view, offer scientists with a way to recognize that an experiment is reliable. That an experiment is reliable is, on Massimi's view, a perspective-independent fact of the matter, and in this way, we can still remain realists with respect to the truth of experimental knowledge claims.

In this way, I then argued, Massimi restricts her perspectivism, since it entails that it is not concerned with how experimental knowledge claims are produced: to ensure realism, she takes the construction of reliable experiments to be free of perspectival influences. On the basis of a suggestion by Giere about how scientists handle incompatibilities between perspectives that are problematic, I then argued that we could in fact allow for perspectives to play a role in both the production and justification of experimental knowledge claims, and this without giving up on

To elaborate this suggestion, I made use of Uljana Feest's work on experimenters' regress situations, which concerns how scientists address such situations in which they are confronted 
with problematic incompatibilities between experimental results. This led me to reconceptualize perspectives as embodied and situated ways of going about in experimental practice, which can become publicly explicated through what I called the process of interpretation. The role played by perspectives on this view is exploratory: they guide scientists in their search for an operationalization of the questions under investigation that can lead them to the production of a reliable experiment. Because of this exploratory characterization, perspectives are historically dynamical entities. The process of interpretation offers scientists a way to explicate the perspective of others, and in doing so, they also gain insight into their own perspective. In this way, they can arrive at a better understanding of how to operationalize, which at the same time also transforms their own perspective.

This view then led me to argue that it is necessary to revise the epistemology of experimentation underlying Massimi's perspectivism. That an experiment is reliable and hence can act as a ground for truth or falsity can only be seen as the result of a whole process, which involves both exploratory and replicating experiments, and interpretation by others. As such, a perspective in itself cannot show a scientist that her experiments are reliable: this necessarily also involves that they are recognized as such by others, which inherently involves scrutiny, criticism and replication.

This view still allows for realism with respect to experimental knowledge claims, I then argued: if scientists are able, through the exploratory interpretative process of experimentation, to construct an experiment that both they and others see as functioning reliably, we can take the knowledge claims that scientists derive from such an experiment to be true, and the entities supposedly manipulated in such an experiment to exist. In this way, we arrive at a perspectivism that can offer us an understanding of the role played by perspectives in both the production and justification of experimental knowledge claims. It can therefore be taken to encompass Massimi's 
perspectivism, but it goes broader in that it allows us to focus in more detail on the practical work that is required to get an experiment to function reliably.

In section 1 I hinted at the possibility that the account presented here of perspectives as primarily performing an exploratory function could equally well shed light on how scientists produce theoretical models of the target systems they are interested in. This is to be expected, for as Giere 2006, p. 49) already pointed out, scientists can only draw conclusions about a target system from experimental data obtained about it by also making use of theoretical models of the system. This suggests that the perspectival exploration of how to experimentally operationalize the questions regarding a target system under investigation will equally well bring scientists to reflect on how to understand and model the target system theoretically. This idea seems to be in line with some of Massimi's recent work on what she calls exploratory or perspectival modeling 2018b 2019b), which concerns how in fields that are conceptually still very open, scientists are able to construct models of target systems they do not yet know to exist, in order to obtain information, constrained by experimental results, about what is possible with regards to such target systems. This idea comes very close to Feest's view of operational analysis as an exploration, in fields that are conceptually still very open, of how a target system can be operationalized in situations where scientists do not yet know whether the phenomena or entities in question exist (see the quote on page 19p. How this link between perspectival exploration in the case of experimentation and in the case of modeling is to be understood exactly, is a question I, for now, have to leave open for further exploration.

Acknowledgements The author would like to thank Michela Massimi, Friedrich Steinle, Marco Giovanelli, Bert Leuridan, Karim Zahidi, Jo Bervoets, Ana-Maria Creţu and the audience of the Edinburgh Graduate Work in Progress seminar for discussions of the ideas contained in this paper. 


\section{References}

Bogen, James and Woodward, James. Saving the phenomena. Philosophical Review 97(3): 305 $-352$.

Chakravartty, Anjan. 2010. Perspectivism, inconsistent models, and contrastive explanations. Studies in History and Philosophy of Science 41: $405-412$.

Collins, Harry. 1985. Changing order. Replication and induction in scientific practice. Chicago: The University of Chicago Press.

Feest, Uljana. 2003. Exploratory experimentation, concept formation, and theory construction in psychology. In: Feest, Uljana \& Steinle, Friedrich (eds.). Scientific concepts and investigative practice. Berlin: de Gruyter.

Feest, Uljana. 2011. What exactly is stabilized when phenomena are stabilized?. Synthese 182: $57-71$.

Feest, Uljana. 2016. The experimenters' regress reconsidered: Replication, tacit knowledge, and the dynamics of knowledge generation. Studies in History and Philosophy of Science 58: $34-$ 45.

Giere, Ron. 2006. Scientific Perspectivism. Chicago: The University of Chicago Press.

Giere, Ron. 2009. Scientific Perspectivism: behind the stage door. Studies in History and Philosophy of Science 40: $221-223$.

Hacking, Ian. 1982. Language, truth and reason. In: Hollis, Martin and Lukes, Steven (eds.). Rationality and relativism. Cambridge: The MIT Press.

Hacking, Ian. 1983. Representing and intervening: Introductory topics in the philosophy of natural science. Cambridge: Cambridge University Press.

Hacking, Ian. 1991. 'Style' for historians and philosophers. Studies in History and Philosophy of Science 23(1): $1-20$. 
Hacking, Ian. 1992. The self-vindication of the laboratory sciences. In: Pickering, Andrew (ed.). Science as practice and culture. Chicago: The University of Chicago Press.

MacFarlane, John. 2005. Making sense of relative truth. Proceedings of the aristotelian society 105(1): $305-323$.

Massimi, Michela. 2012. Scientific Perspectivism and its Foes. Philosophica 84: 25 - 52.

Massimi, Michela. 2015. Walking the line: Kuhn between realism and relativism. In: Devlin, William J. \& Bokulich, Alisa (eds.). Kuhn's Structure of Scientific Revolutions - 50 years on. Heidelberg: Springer.

Massimi, Michela. 2016. Three Tales of Scientific Success. Philosophy of Science 83: $757-767$.

Massimi, Michela. 2018. Four Kinds of Perspectival Truth. Philosophy and Phenomenological Research XCVI: $342-359$.

Massimi, Michela. 2018b. Perspectival modeling. Philosophy of Science 85: 335 - 359.

Massimi, Michela. 2019a. Realism, perspectivism, and disagreement in science. Synthese. https: //doi.org/10.1007/s11229-019-02500-6.

Massimi, Michela. 2019b. Two kinds of exploratory models. Philosophy of Science 86: $869-881$. Massimi, Michela \& McCoy, Casey D. 2019. Understanding Perspectivism. Scientific Challenges and Methodological Prospects. New York: Routledge.

Morrison, Margaret. 2011. One phenomenon, many models: Inconsistency and complementarity. Studies in History and Philosophy of Science 42: 342 - 351.

Rouse, Joseph. 1987. Knowledge and power. Towards a political philosophy of science. Ithaca: Cornell University Press.

Rueger, Alexander. 2005. Perspectival Models and Theory Unification. British Journal for Philosophy of Science 56: $579-594$.

Rueger, Alexander. 2016. Perspectival Realism and Incompatible Models. Axiomathes 26: $401-$ 410. 
Schickore, Jutta. 2016. "Exploratory experimentation" as a probe into the relation between historiography and philosophy of science. Studies in History and Philosophy of Science 55: 20 $-26$.

Soler, Lèna. 2011. Tacit aspects of experimental practices: analytical tools and epistemological consequences. European Journal for Philosophy of Science 1: 393 - 433.

Sosa, Ernest. (1991). Knowledge in perspective. Selected essays in epistemology. Cambridge: Cambridge University Press.

Steinle, Friedrich. 1997. Entering new fields: Exploratory uses of experimentation. Philosophy of Science 64: S65 - S74.

Steinle, Friedrich. 2016. Exploratory experiments: Ampére, Faraday, and the origins of electrodynamics. Pittsburgh: University of Pittsburgh Press.

Teller, Paul. 2001. The Twilight of the Perfect Model Model. Erkenntnis 55: 393 - 415.

Turner, Stephen. 2014. Understanding the Tacit. New York: Routledge.

van Fraassen, Bas. 2008. Scientific Representation: Paradoxes of Perspective. Oxford: Oxford University Press.

Vogel, Jonathan. 2000. Reliabilism leveled. The Journal of Philosophy 97(11): $602-623$.

Vogel, Jonathan. 2008. Epistemic Bootstrapping. The Journal of Philosophy 105(9): $518-539$. 